\title{
Synaptic Sites on Reinnervated Nerve Cells Visualized at Two Different Times in Living Mice
}

\author{
D. Purves and J. W. Lichtman \\ Department of Anatomy and Neurobiology, Washington University School of Medicine, St. Louis, Missouri 63110
}

\begin{abstract}
Synaptic boutons on the surface of identified autonomic ganglion cells were visualized by methylene blue staining at intervals of 1-2 months following denervation to assess whether regenerating axon terminals reoccupy original synaptic sites. The distribution of synapses observed on the same neuronal cell bodies was almost always different in appearance after reinnervation. These results are at odds with the conclusions of earlier workers, who have argued that mammalian neurons bear a fixed number of synaptic sites, which are reoccupied during reinnervation.
\end{abstract}

The stability of synapses in the mammalian nervous system remains a largely unresolved issue; it is an important one because the relative permanence of synaptic sites bears on the cellular basis of long-term ncural change. Electron-microscopical analysis of reinnervation in both an autonomic ganglion and the central nervous system of the rat has suggested that mammalian neurons have a fixed number of postsynaptic sites and that no new postsynaptic sites are formed during reinnervation (Raisman and Field, 1973; Raisman et al., 1974). We have reinvestigated the permanence of synaptic sites in an autonomic ganglion more directly, taking advantage of the fact that it is possible to locate and study the same nerve cells in a living mammal at 2 different times, separated by an arbitrarily long interval (Purves and Hadley, 1985; Purves et al., 1986a b). In the studies reported here, we identified synapses on the surface of mouse submandibular ganglion cells by staining them with methylene blue; after intervals of 1-2 months, the same cells were located and their innervation stained once again.

\section{Materials and Methods}

Young adult male mice (CF1 strain; 25-30 gm) were anesthetized with chloral hydrate and placed on a focusing stage so that the animal could be viewed under either a dissecting or a compound microscope (see Purves et al., 1986a). A midline incision was made in the neck and the parasympathetic ganglia along the salivary ducts to the sublingual and submaxillary glands were exposed (Fig. 1). There are usually 3 or 4 of these ganglia, which, together with several groups of ganglion cells lying more proximally (Lichtman, 1977), supply the parasympathetic innervation to the submandibular and sublingual salivary glands.

In order to visualize individual neurons, a small, optically flat mirror was placed underneath the ganglion so that the cells of interest lay above the reflecting surface (Fig. 2). The ganglion was then illuminated through

Received July 25, 1986; revised Oct. 15, 1986; accepted Oct. 24, 1986.

This work was supported by grants from the NIH, the MDA, a Research Development Award from the McKnight Foundation to J.W.L., and the Jacob Javits Center for Neuroscience at Washington University.

Correspondence should be addressed to Dr. Dale Purves, Department of Anatomy and Neurobiology, Washington University School of Medicine, 660 Euclid Avenue, St. Louis, MO 63110.

Copyright (C) 1987 Society for Neuroscience $0270-6474 / 87 / 051492-06 \$ 02.00 / 0$ the microscope objective using a conventional I eitz epi-illumination system. Light reflecting off the surface of the mirror revealed superficial ganglion cells in considerable detail (see Fig. 3).

Neurons on the surface of one of the several ganglia along the ducts were photographed on $35 \mathrm{~mm}$ film to allow subsequent identification of the same cells. Synaptic boutons on these cells are located primarily on the cell body since these neurons lack dendrites (see Fig. 3, inset) and invariably receive dominant synaptic input from a single axon (Snider, in press; see also Lichtman, 1977, 1980). To visualize synaptic boutons we used a modification of the classical methylene blue staining procedure (Polyak, 1941). A $1 \mathrm{ml}$ amount of methylene blue stock solution (Sigma; $2 \mathrm{mg} / \mathrm{ml}$ in normal saline) was freshly added to $10 \mathrm{ml}$ of lactated Ringer's fluid (Travenol), together with $0.5 \mathrm{ml}$ of $0.1 \mathrm{M}$ HEPES buffer and $50 \mathrm{mg}$ of ascorbic acid; the solution was adjusted to pH 6.5 before use. In order to stain the houtons, the solution was perfused through the wound at a rate of about $1 \mathrm{ml} / \mathrm{min}$. The sterile plastic tubing carrying the staining mixture was illuminated by a light pipe (6-mm-diameter, $150 \mathrm{~W}$ projector lamp) to bleach a fraction of the blue dye to its leucobase form (the moiety that presumably enters the cells; see Polyak, 1941). During staining, the ganglion was viewed with green light $(546 \mathrm{~nm})$ to slow the photodamage produced by the dye (methylene blue has an absorbance maximum of about $620 \mathrm{~nm}$ and causes swelling of stained endings; this phenomenon is accelerated by strong illumination). Within 1-2 min, stained boutons began to appear on ganglion cells. As soon as boutons could be visualized on most cells, the staining solution was flushed out with lactated Ringer's fluid for 3$4 \mathrm{~min}$. Additional photographs were then taken to record the configuration of synaptic boutons on the cells previously chosen for study (see Figs. 4 and 5). In one series of experiments, the preganglionic nerve was crushed about $1-2 \mathrm{~mm}$ proximal to the ganglia at the time of operation (see Figs. 1, 2); in another series, the nerve was left intact. Following an additional $5 \mathrm{~min}$ washout with lactated Ringer's fluid, the wound was closed, and the animals were revived and returned to their cages in the animal quarters. After an interval of 1-2 months, this procedure was repeated exactly and the neurons initially studied were located for staining a second time. Photographs were taken at several different focal planes and the configuration of synaptic boutons on the identified ganglion cells compared with that in the initial pictures.

\section{Results}

Synaptic boutons on identified ganglion cells were differently arranged at the second viewing in mice whose preganglionic nerves had been crushed at the time of the initial operation (Fig. 4). A total of 77 different neurons were identified at the 2 viewings ( 9 animals). On the majority of these cells the patterns were clearly different after reinnervation; on only 1 cell was the pattern similar. Comparisons over this interval revealed many instances in which boutons were seen at sites where there were no boutons at the initial viewing, and other instances in which boutons were missing from sites where they had been observed initially. Although synaptic boutons were often found on the same general region of cell surface after reinnervation, the detailed configuration at the 2 viewings was almost always different. These observations suggest that when axons find their way 


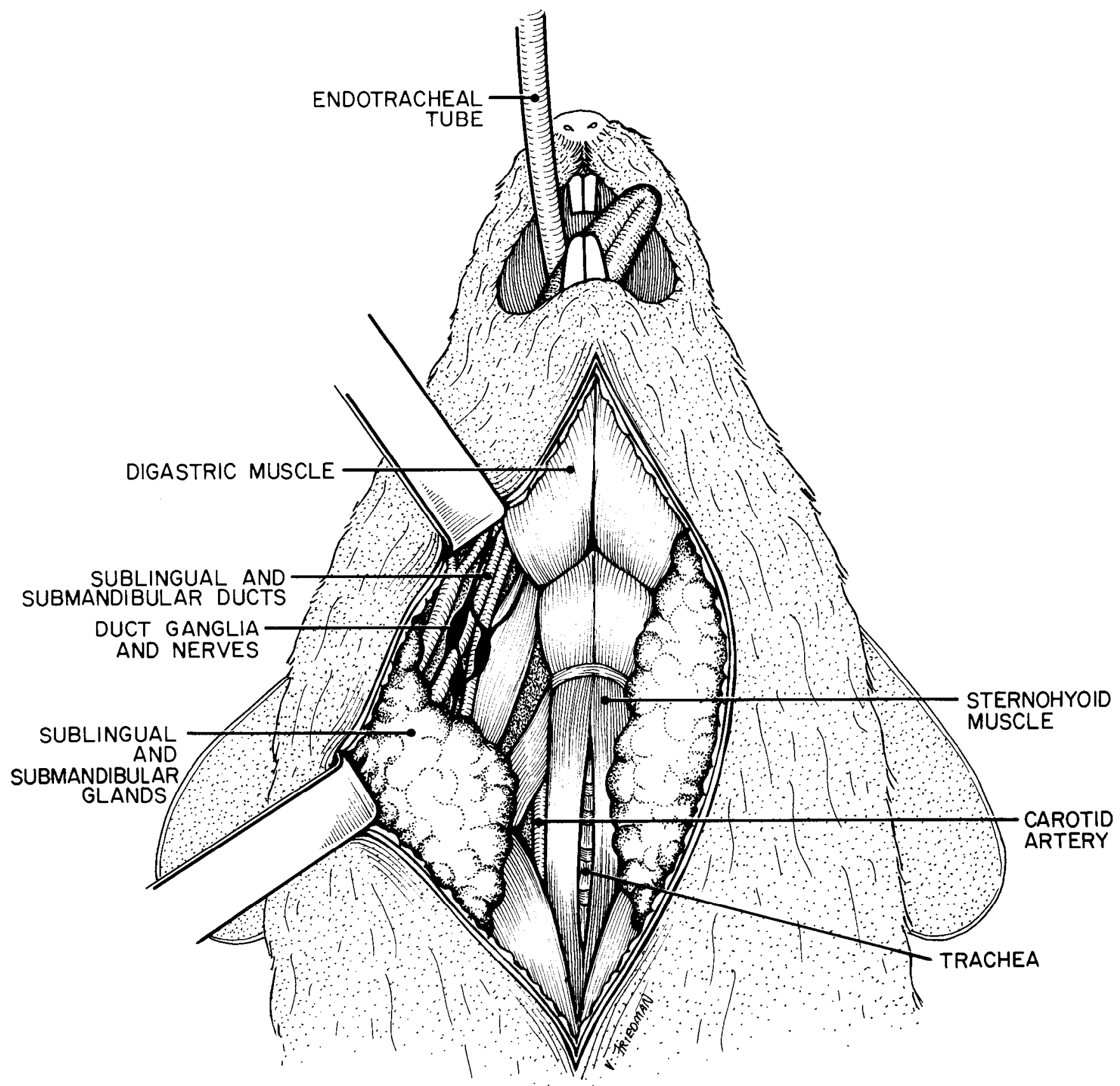

Figure 1. Diagram of the submandibular and sublingual salivary duct ganglia after surgical exposure in the mousè. Three or 4 ganglia can usually be identified along these ducts; the ganglion that we used most frequently was the one along the lateral (sublingual) salivary duct. The arrangement of ganglia shown here is typical, but by no means invariant.

back to the postsynaptic cell, they do not innervate the same synaptic sites.

In order to assess whether this apparent rearrangement of synaptic sites during reinnervation was the result of axonal interruption and misrouting at the site of the nerve crush, a second series of experiments was conducted in which the nerve was left intact. Our assumption in these experiments was that nerve terminals locally damaged by the staining procedure would rapidly regrow with a minimum of anatomical disruption, thus maximizing the opportunity for reoccupation of former synaptic sites. The presumption of local damage was based on our ob- servations that the nerve terminals begin to swell within several minutes of methykene blue staining, and that postsynaptic potentials recorded intracellularly during ganglion cell staining rapidly disappear.

The results of the experiments in which the nerve was left intact were similar to those observed after denervation (Fig. 5). A total of 121 neurons from 21 mice was examined. Again, on the majority of these cells, synaptic boutons visualized after a period of 1-2 months were configured differently than at the time of initial staining. On none of the 121 cells examined were the patterns of synaptic boutons clearly similar. 


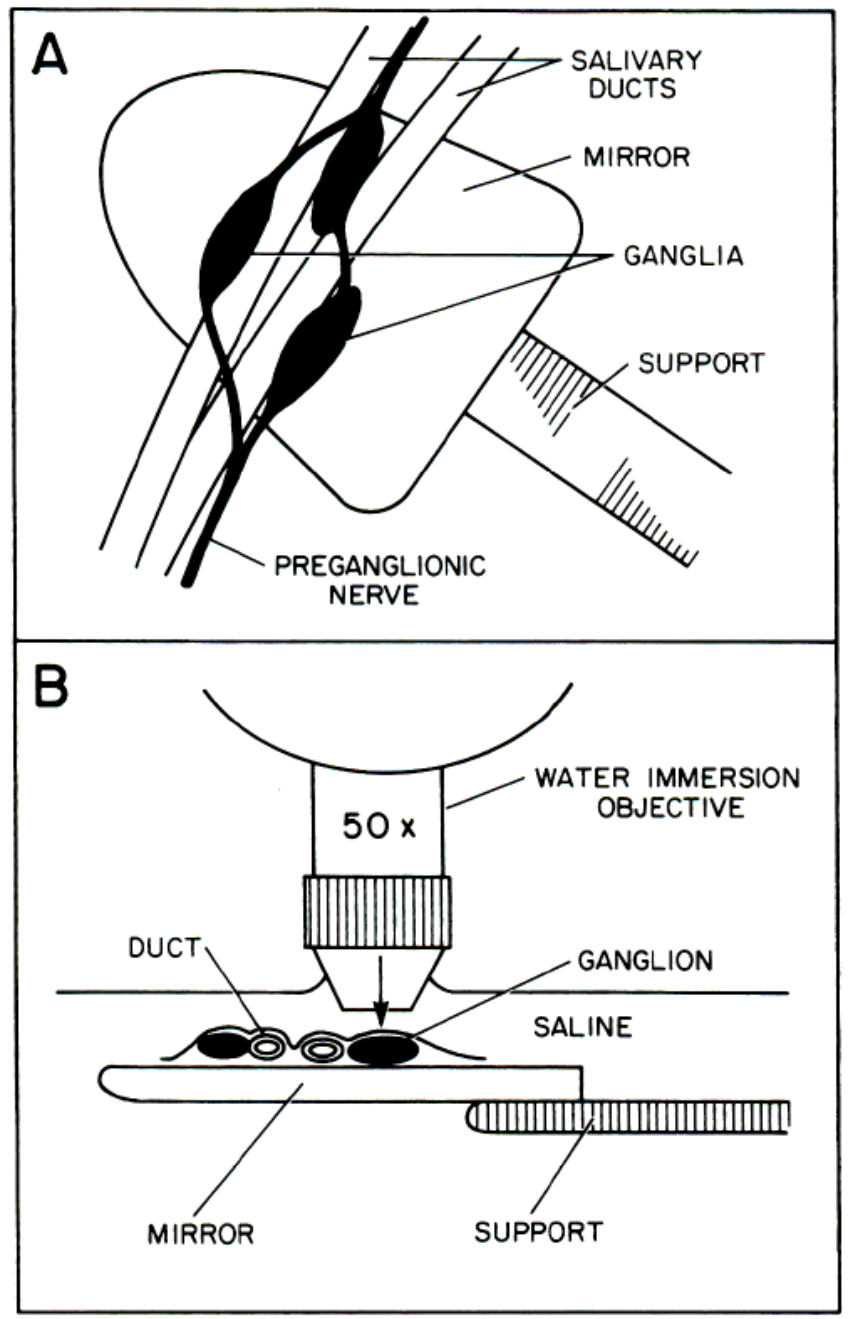

Figure 2. Method of visualizing surface neurons in salivary duct ganglia. A small mirror is placed under the ganglion to reflect light focused through the microscope objective. $A$, View looking down on the mirror, as the preparation would be seen under low power. $B$, The arrangement in side view, with a $50 \times$ objective in place. Arrow indicates direction of illumination.

\section{Discussion}

The idea that synaptic boutons on mammalian nerve cells represent a fixed number of stable sites is based largely on the observation that vacated synaptic sites, identified by postsynaptic thickenings, become more abundant after denervation and then, over a period of weeks to months, diminish in parallel with the regeneration of synapses (Raisman and Field, 1973; Raisman et al., 1974). There are, however, several uncertainties about this interpretation. First, the ultrastructural criteria for identifying a vacated synaptic site and its reoccupation are to some degree subjective. Second, even if correctly identified, the disappearance and reappearance of occupied postsynaptic specializations do not necessarily indicate that the same sites have been reinnervated. The observations we report here, in which

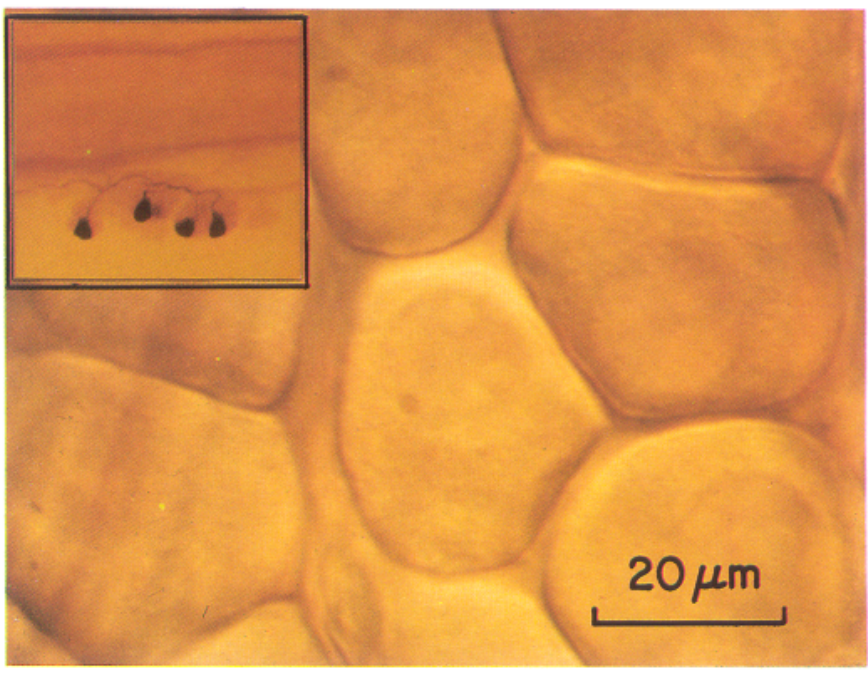

Figure 3. Surface view of neurons in a salivary duct ganglion in situ. The method of illumination allows neurons to be recognized as individual cells in the living animal. Inset, Low-power view of a fixed preparation after 4 cells had been stained by intracellular injection of HRP (kindly provided by W. Snider). The neurons lack dendrites and therefore receive synaptic contacts directly on the cell body and proximal axon. One of the submandibular salivary ducts lies just above this small group of cells.

synaptic boutons on the same cell were visualized at 2 different times, argue that synaptic sites on reinnervated mammalian nerve cells are not strongly fixed since the pattern of synaptic contacts on the same cell over intervals of several weeks was different.

There are, however, some uncertainties in our own observations. First, it is difficult to examine exactly the same focal plane at 2 different times. Although we photographed several planes in order to include all surface boutons on identified ganglion cells, some apparent differences in the configuration of identical synaptic arrangements might be expected on this basis. A second problem is that methylene blue may not always stain the full complement of synaptic boutons on each cell. One might, therefore, interpret the staining of different subpopulations of an identical set of boutons as indicating different synaptic sets. This variability might also account for some of the differences we observed. Finally, because methylene blue is toxic, we could not assess the normal stability of synaptic sites with this technique (see, however, Purves et al., 1986b). Whether or not synaptic sites are normally in flux on these cells is obviously relevant to the interpretation of the present observations.

Because of these ambiguities, our results must be regarded with caution. Nevertheless, the outcome for the large number of reinnervated cells that we examined was quite consistent. A conservative view would be that the results we describe should reopen the important question of the permanence of synaptic sites on mammalian nerve cells. At face value, our results suggest that synapses on mature neurons may be considerably more dynamic than was previously imagined. 
VENTRAL SIDE

DORSAL SIDE

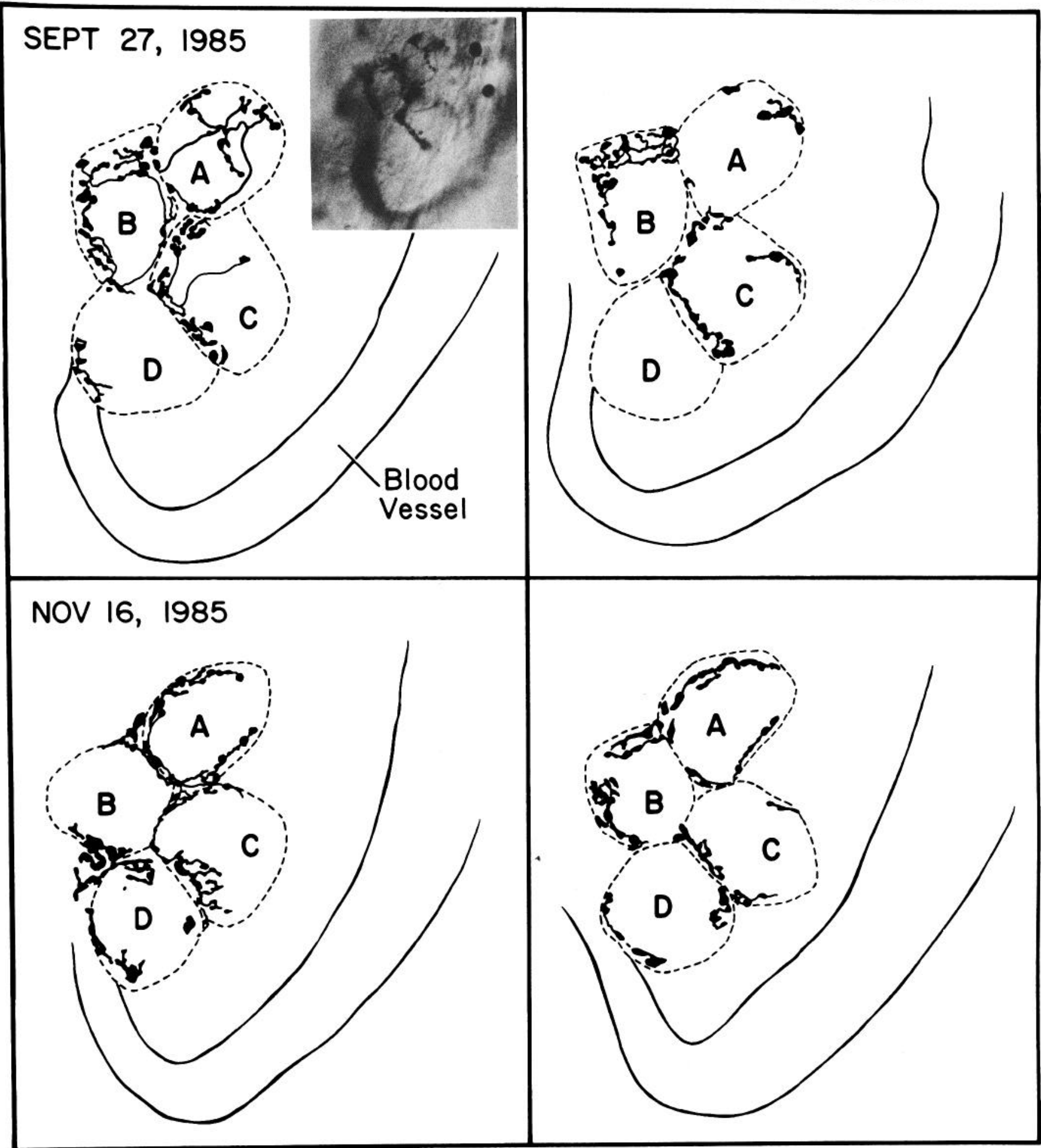

Figure 4. Camera lucida drawings of the synaptic boutons on the ventral and dorsal surfaces of a cluster of ganglion cells stained with methylene blue initially (top panels) and 2 months later (bottom panels). Inset, Photograph of the neurons at the first viewing (ventral side). The cells were relocated by their size, position, and arrangement, and by local landmarks such as blood vessels. In the vast majority of preparations, the location of synaptic boutons on ganglion cell surfaces was different after reinnervation (see also Fig. 5). 

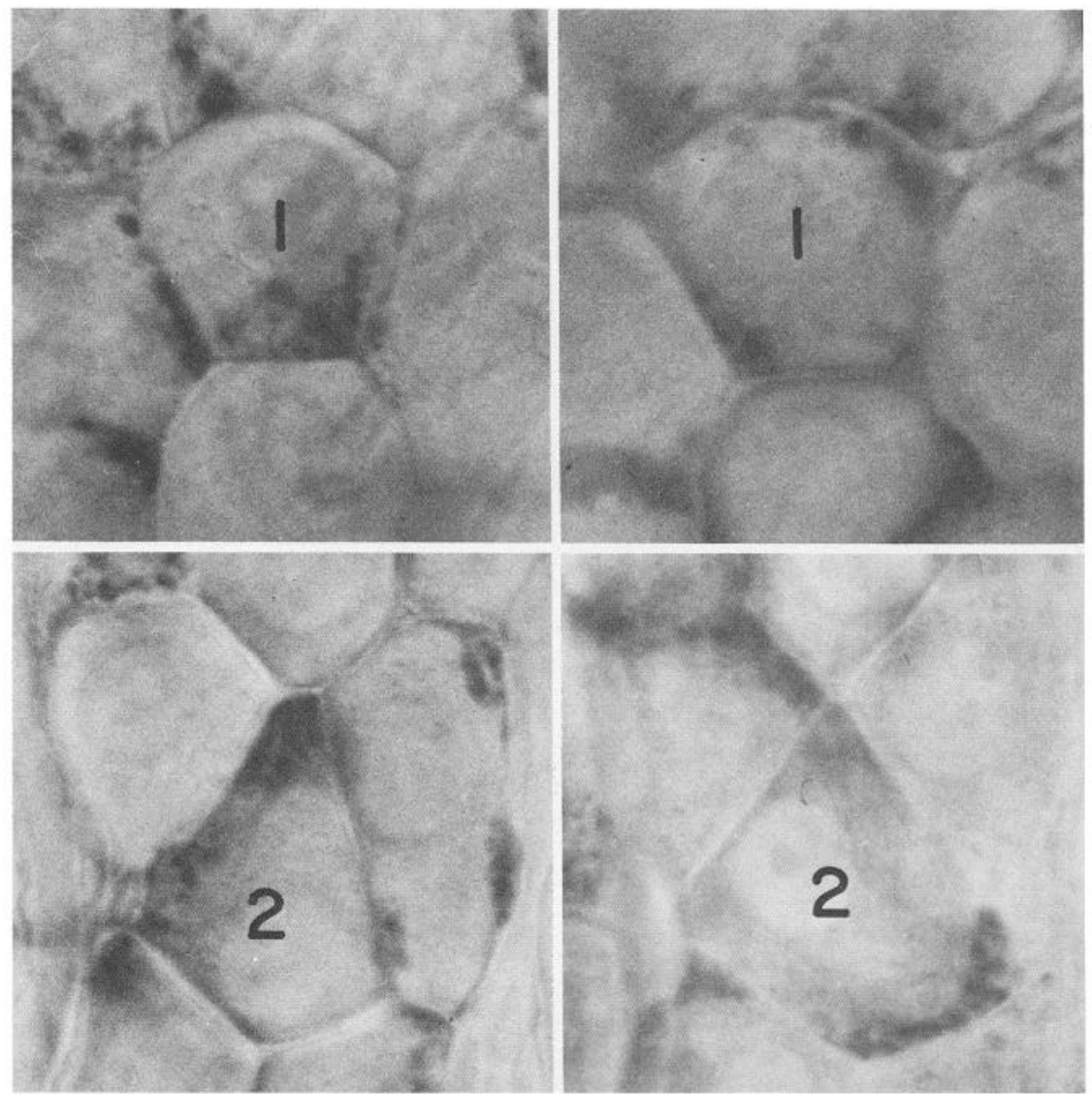

Figure 5. Synaptic boutons on representative salivary duct ganglion cells stained at an interval of 1-2 months; in these experiments the preganglionic nerve was left intact. The numbers indicate corresponding neurons at the beginning and end of this period. The results were similar to those seen after denervation, i.e., patterns of synaptic contacts were always different at the end of this interval. Calibration bar applies to all panels.
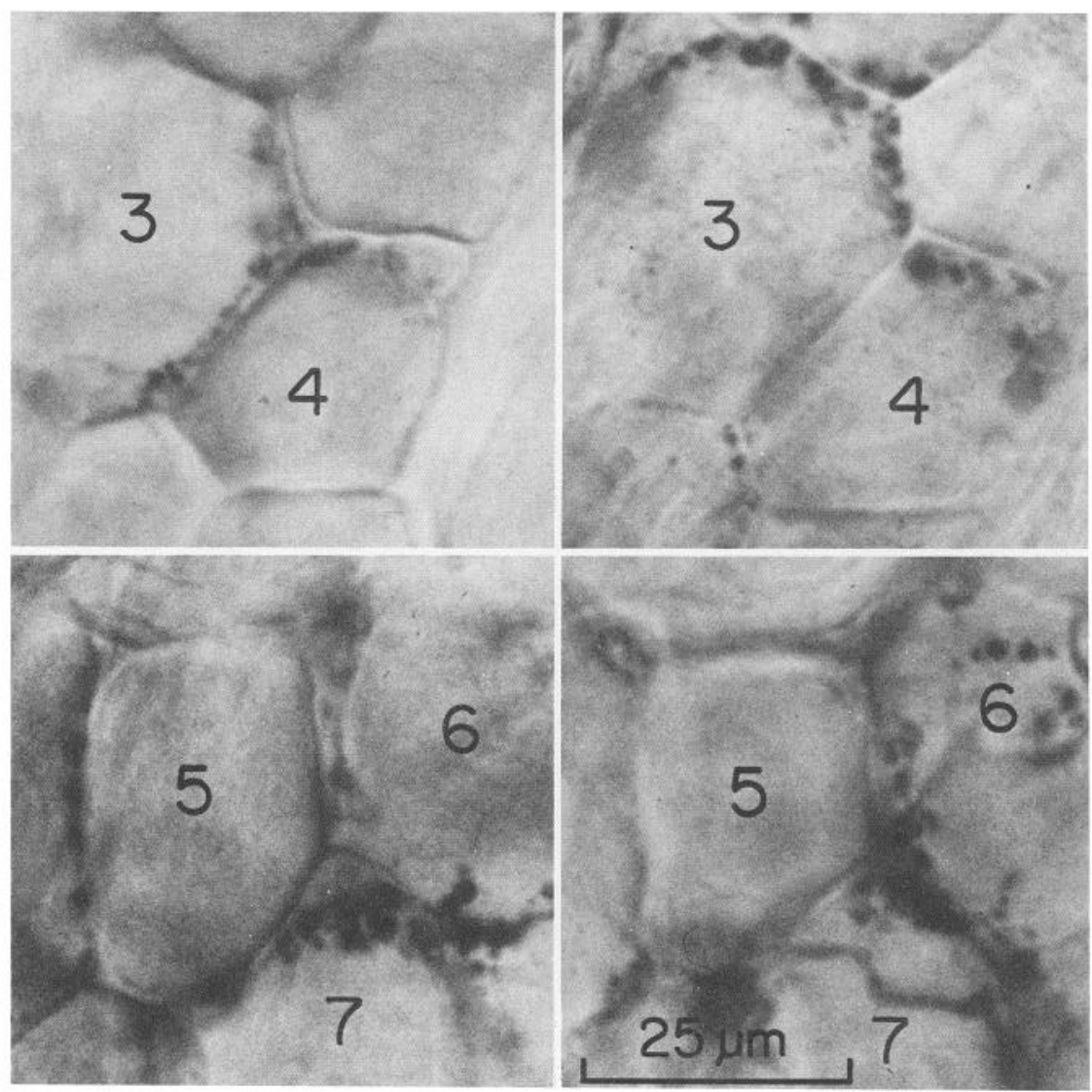


\section{References}

Lichtman, J. W. (1977) The reorganization of synaptic connexions in the rat submandibular ganglion during post-natal development. J. Physiol. (Lond.) 273: 155-177.

Lichtman, J. W. (1980) On the predominantly single innervation of submandibular ganglion cells in the rat. J. Physiol. (Lond.) 302: 121130.

Polyak, S. L. (1941) The Retina, U. Chicago P., Chicago.

Purves, D., and R. D. Hadley (1985) Changes in the dendritic branching of adult mammalian neurones revealed by repeated imaging in situ. Nature 315: 404-406.

Purves, D., R. D. Hadley, and J. Voyvodic (1986a) Dynamic changes in the dendritic geometry of individual neurons visualized over periods of up to three months in the superior cervical ganglion of living mice. J. Neurosci. 6: 1051-1060.
Purves, D., H. Yawo, and J. Voyvodic (1986b) Systemic administration of a fluorescent dye visualizes living nerve terminals in a mammalian autonomic ganglion. Soc. Neurosci. Abstr. 12: 390.

Raisman, G., and P. M. Field (1973) A quantitative investigation of the development of collateral reinnervation after partial deafferentiation of the septal nuclei. Brain Res. 50: 241-264.

Raisman, G., P. M. Field, A. J. C. Ostberg, L. L. Iverson, and R. E. Zigmon (1974) A quantitative ultrastructural and biochemical analysis of the process of reinnervation of the superior cervical ganglion in the adult rat. Brain Res. 71: 1-16.

Snider, W. D. (1987) The dendritic complexity and innervation of submandibular neurons in five species of mammals. J. Neurosci. (in press). 